\title{
The Lipid Organisation in Human Stratum Corneum and Model Systems
}

\author{
Joke A. Bouwstra* and Gert S. Gooris \\ Leiden/Amsterdam Center for Drug Research, Gorlaeus Laboratories, Leiden University, P.O. Box 9502, 2300 RA \\ Leiden, The Netherlands
}

\begin{abstract}
The primary function of the skin is to act as a barrier against unwanted influences from the environment. The barrier function of the skin is located in the superficial of the skin, the stratum corneum. The stratum corneum consists of dead cells filled with keratin and water, which are embedded in lipid regions. The lipid regions are the only continuous structure in the stratum corneum. For this reason the lipid regions are considered to be very important for the barrier function. The main lipid classes are ceramides, cholesterol and free fatty acids. In this paper the lipid organisation in human stratum corneum is reviewed. In addition, the role the various lipid classes play in the lipid organisation will be discussed using mixtures prepared from either native human ceramides or synthetic ceramides. Finally a model, referred to as the stratum corneum substitute, is described in which the lipid organisation, composition and barrier function can be examined.
\end{abstract}

Keywords: Lipids, ceramides, X-ray diffraction, lamellar phase, lateral packing, barrier function.

\section{LIPID COMPOSITION IN HUMAN STRATUM CORNEUM}

The upper layer of the skin is formed by a transparent 10$15 \mu \mathrm{m}$ thick layer, which consists of dead cells, corneocytes embedded in lipid regions. This layer is responsible for the primary barrier of the skin. The corneocytes are flat dead cells filled with keratin filaments, water and the natural moisturising factor [1]. The corneocytes are surrounded by a densely crosslinked protein layer, referred to as the cell envelope. A monolayer of lipids is chemically linked to this densely packed cell envelope [2]. This lipid monolayer has a crucial role in the stratum corneum as it serves as an interface between the hydrophilic corneocytes and the lipophilic extracellular lipid matrix. Furthermore, corneodesmosomes interconnect the corneocytes and play an important role in the stratum corneum cohesion. The loss of cells from the stratum corneum is compensated by the cell growth in the innermost layer of the epidermis, the stratum basale. In this way the thickness of the epidermis remains approximately constant.

In human stratum corneum the major lipid classes $[3,4]$ are ceramides (CERs), cholesterol (CHOL) and saturated long chain free fatty acids (FFAs). The ratio between these lipid classes is approximately equimolar [5]. Low levels of other lipid classes are also present, such as cholesterol sulphate, glucosylceramides and cholesterol esters. In human stratum corneum 11 subclasses of CERs have been identified [6-9]. The CERs head groups are very small and contain several functional groups that can form lateral hydrogen bonds with adjacent molecules. The CERs differ by the head-group architecture and fatty acid chain length. The base consists of either a sphingosine (S), phytosphingosine (P),

\footnotetext{
*Address correspondence to this author at the Leiden/Amsterdam Center for Drug Research, Gorlaeus Laboratories, Leiden University, P.O. Box 9502, 2300 RA Leiden, The Netherlands; Tel: 31-71-5274208; Fax: 31-715274565; E-mail: bouwstra@chem.leidenuniv.nl
}

6-hydroxysphingosine (H) or dihydrosphingosine (D). Chemically linked to the base is a fatty acid, with again three variations in structure, namely a non-hydroxylated fatty acid $(\mathrm{N})$, an $\alpha$-hydroxy fatty acid (A) or an $\omega$-hydroxy fatty acid. Both, the base and the fatty acid exhibit a chain-length distribution. Combining the four bases with the $\mathrm{N}$ and $\mathrm{A}$ fatty acids results in 8 CER structures, referred to as AS, AP, $\mathrm{AH}, \mathrm{AD}$ and NS, NP, NH and ND. The $\omega$-hydroxy fatty acids have a very long chain length up to 34 carbon atoms. Furthermore, a linoleic acid is ester linked to this $\omega$-hydroxy group (EO), which results in 3 additional very exceptional CERs, referred to EOS, EOH and EOP [10].

\section{LIPID ORGANISATION IN HUMAN STRATUM CORNEUM}

As far as the lipid organisation is concerned, the lateral packing as well as the lamellar phases are considered to be important for the skin barrier function. Already in 1991, the lamellar phases in human stratum corneum were identified. Small angle X-ray diffraction studies revealed the presence of two lamellar phases in human stratum corneum: one lamellar phase with a periodicity of approximately $6 \mathrm{~nm}$ referred to as SPP (= short periodicity phase), and the long periodicity phase (LPP) with a periodicity of approximately $13 \mathrm{~nm}$ [11] (see Fig. 1). The LPP is present in all species examined until now, and has a very characteristic molecular organisation. For this reason it has been suggested that the presence of this phase plays an important role in skin barrier function.

When filling a three-dimensional space, the lipid organisation in the plane perpendicular to the direction in which the lamellar phases are defined should also be characterised. In this plane, parallel to the basal plane of the lamellae, the lipids are arranged in either a liquid phase, a hexagonal phase or an orthorhombic lateral packing. The orthorhombic lateral packing is very densely packed. The neighboring molecular distance is not equal in all directions. 


\section{Lamellar phases: X-ray diffraction}
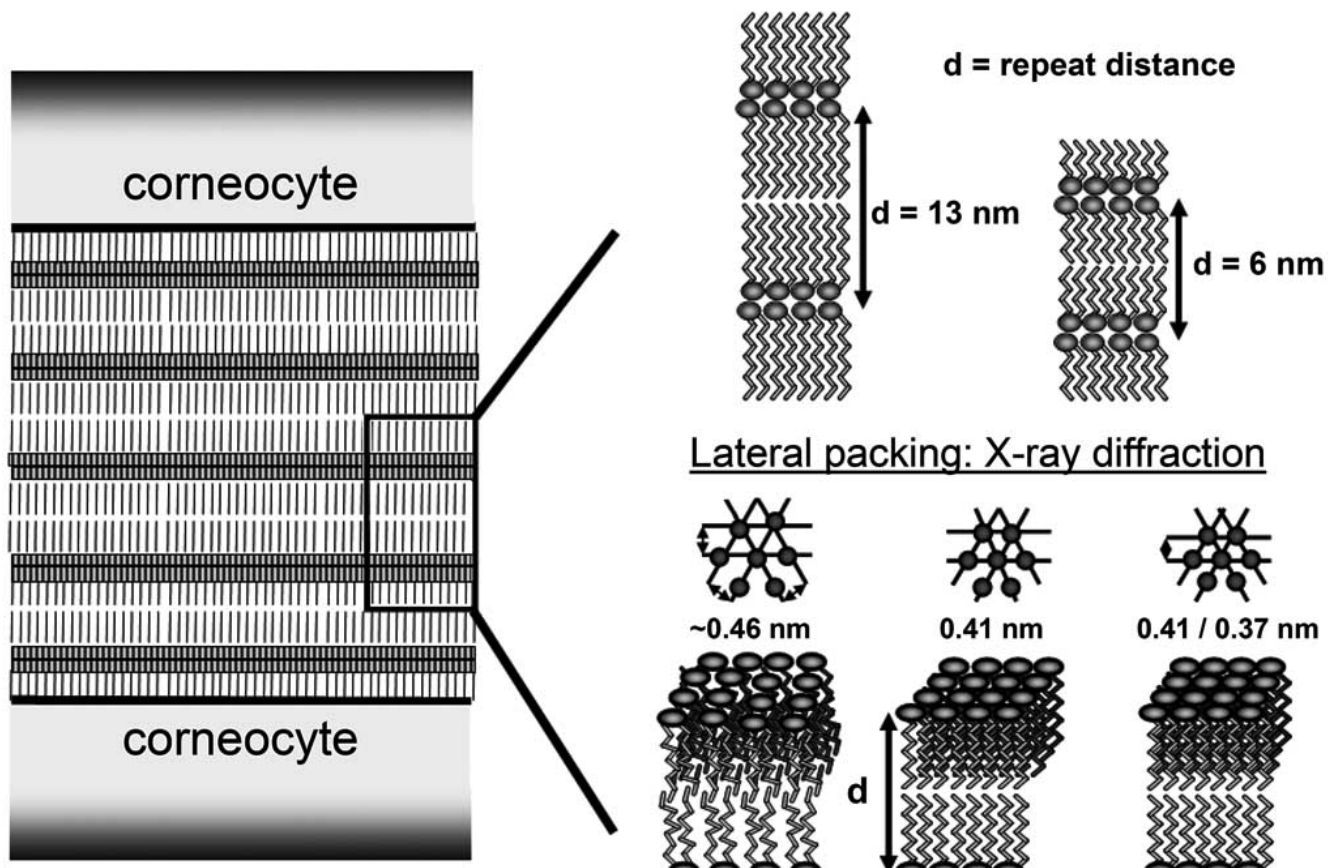

\section{Lateral packing: X-ray diffraction}
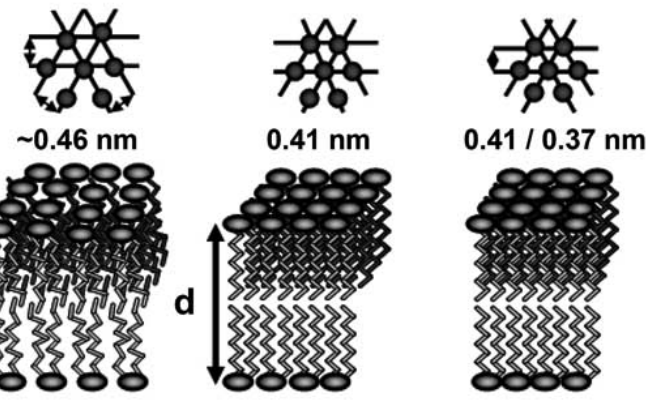

high transport medium transport

low transport liquid hexagonal orthorhombic

Fig. (1). A schematic drawing of the lamellar phases in human stratum corneum. Between the corneocytes the lipids are organised in two lamellar phases with repeat distances of 6 and $13 \mathrm{~nm}$. However, these lamellar phases fill the three dimensional space only in one direction. In the plane perpendicular to the lamellar phases, another organisation can be identified, namely the lateral packing. This lateral packing is either liquid, hexagonal or orthorhombic. The orthorhombic lateral packing is dominantly present in human stratum corneum.

In one direction, the lipids are more densely packed, resulting in two strong diffraction rings at 0.37 and $0.41 \mathrm{~nm}$ spacing (see Fig. 1).

Wide angle X-ray diffraction studies revealed the presence of an orthorhombic lateral packing in human stratum corneum [12-14]. However, whether a hexagonal phase coexists with the orthorhombic lateral packing remained unclear, as the characteristic $0.41 \mathrm{~nm}$ spacing characteristic for the hexagonal phase is at the same position as the strong intensity ring in the diffraction patterns of the orthorhombic lateral packing. However, using electron diffraction single crystals are exposed to the electron beam, which makes it easier to distinguish the two phases. Electron diffraction revealed the presence of an hexagonal lateral packing $[15,16]$. Studies performed as function of depth in stratum corneum showed that the hexagonal lateral packing is more prominently present in the top layers than in the deeper layers of human stratum corneum. The presence of an orthorhombic phase is also observed with infrared spectroscopy and indicates that the lipids within the lamellae are very densely packed [17]. Very recently it has been shown that there is a correlation in the degree the orthorhombic lateral packing is present in stratum corneum and the trans-epidermal water loss values [18]. The latter is a measure for the skin barrier. This demonstrates that the orthorhombic lateral packing plays indeed a role in the skin barrier function. It remained unclear whether a liquid phase coexisted with the orthorhombic lateral packing, as the broad reflection of the liquid phase in the diffraction pattern was obscured by the reflections based on soft keratin present in the corneocytes. Frequently some CHOL phase separates from the lamellar phases $[12,13]$.

\section{RELATION BETWEEN LIPID COMPOSITION AND ORGANISATION}

Information on the relationship between lipid organization and lipid composition is of great importance to unravel the mechanism controlling the skin barrier function of normal and diseased skin. Due to the complexity of the native stratum corneum, it is impossible to modulate systematically lipid composition in the stratum corneum. Especially when one is interested in the relationship between lipid composition and lipid organisation, the use of lipid mixtures isolated from stratum corneum, offers an attractive alternative. In this paper we will focus on the phase behaviour studies performed with human CERs.

When using isolated human CERs and CHOL only, the SPP and LPP with periodicities of 5.4 and $12.8 \mathrm{~nm}$ respectively, were formed in the lipid mixtures mimicking the lamellar phases in human stratum corneum already very closely (see Fig. 2) [19]. This demonstrates that no proteins are required for the formation of these lamellar phases and that in the absence of FFA, the LPP is already formed. The lamellar phases remained unchanged over a wide range of 
CHOL:CER molar ratios (between 0.2 and 1). This indicates that the formation of the lamellar phases is insensitive towards changes in CHOL:CER molar ratio. At a CHOL:CER molar ratio higher than 0.6 , the presence of crystalline $\mathrm{CHOL}$ is observed suggesting that the lamellae are already saturated with CHOL at this molar ratio. This demonstrates that in the in vivo situation a variation in CHOL:CER molar ratio will not lead to a substantial change in lipid phase behavior, but that CHOL will form separate crystalline domains when exceeding the amount necessary to saturate the lamellae. When focusing on the lateral packing, an orthorhombic packing is only formed in the presence of FFA. Furthermore, in the presence of FFA the LPP and SPP were present in the lipid mixtures with repeat distances of 13.0 and $5.5 \mathrm{~nm}$, respectively, demonstrating that CHOL:CER:FFA mixture mimics closely the lipid organisation in intact human stratum corneum $[19,20]$. When using short chain FFA with predominantly C16 and C18 chain length, no orthorhombic lateral packing was detected in CHOL:CER:FFA mixtures [21]. In the presence of long-chain FFAs, besides an orthorhombic also a liquid packing was formed in the lipid mixtures, as the broad reflection at $0.46 \mathrm{~nm}$ (indicative for a liquid phase) was clearly observed in the diffraction pattern.

\section{lipid composition and organisation}

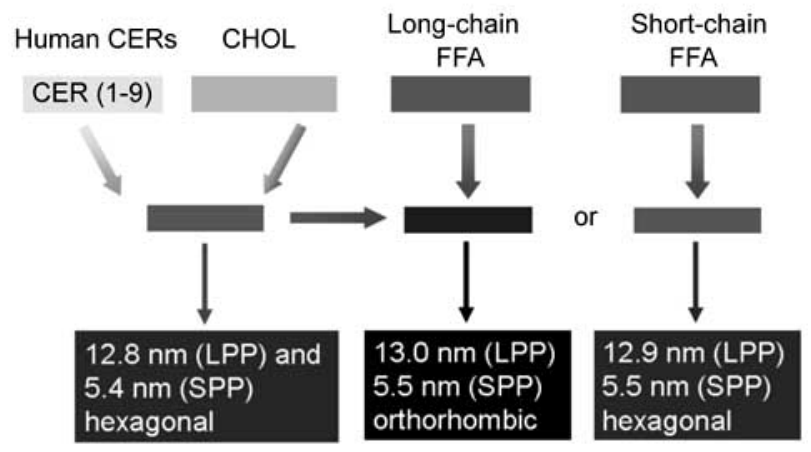

Fig. (2). The relationship between lipid composition and lipid organisation. CHOL and CER are very crucial for the formation of the two lamellar phases, while FFA induce the formation of an orthorhombic lateral packing.

In diseased or dry skin often the lipid composition is different from that in healthy subjects. For example, the levels of EOS and NP are both reduced in stratum corneum of psoriasis skin [22], while the fatty acid levels in stratum corneum of lamellar ichthyosis skin is drastically diminished [23]. Consequently, the lipid organisation may also change: phase studies with lipid mixtures demonstrated that a reduction in EOS level reduces the formation of the LPP, while a reduction in FFA will decrease the population of lipids forming an orthorhombic lateral packing. These findings correlated excellently with ex vivo studies. In stratum corneum of lamellar ichthyosis patients the formation of an orthorhombic lateral packing is strongly reduced, while a reduction in EOS level in normal and dry stratum corneum is correlated with a reduction in the formation of the LPP $[16,24]$. These results demonstrate that lipid mixtures prepared with isolated CER are an excellent tool to provide information on the relation between lipid organisation and composition relevant for the changes in lipid organisation of diseased skin.

\section{LIPID MIXTURES BASED ON SYNTHETIC CERS}

In contrast to the previous investigation, in which solely mixtures prepared with isolated CERs with a variation in chain length were studied, in a more recent study [25] the lipid organisation in mixtures prepared with synthetic CERs with defined acyl chain length was examined. The CER included in these mixtures are EOS (C30), NS (C24), NP (C16 and C24), AS (C24) and AP (C24). The lipid organisation in equimolar mixtures of $\mathrm{CHOL}$, synthetic CERs and FFAs closely resembles that in stratum corneum, as both LPP (12.2. $\mathrm{nm})$ and SPP $(5.4 \mathrm{~nm})$ are present and the lateral packing of the lipids is orthorhombic. Interestingly, in these mixtures FFAs are required for proper lipid organisation, as only in their presence a dominant formation of the LPP could be detected. No data are available yet, whether in these mixtures CER and FFA participate in one orthorhombic lattice. This is of interest as a reduction in chain length variation often reduces the mixing properties. Very interestingly, the mixing properties of the synthetic CERs, CHOL, FFA mixtures mimicked closely that of mixtures prepared from isolated CERs, most probably due to the fatty acid chain length variation [26].

As it is of interest not only to study the relation between lipid composition and organisation, but also the effect of lipid composition on the barrier function, a stratum corneum lipid membrane has been developed mimicking very closely the lipid organisation and orientation in stratum corneum. This lipid membrane is supported by a porous membrane, and is referred to as the stratum corneum substitute (SCS). The SCS is designed in such a way that after preparation it can be fixed in a flow-through diffusion cell to study its barrier properties (see Fig. 3A). These properties were evaluated in a series of in vitro passive diffusion studies, using three structurally related compounds, namely $\mathrm{p}$ aminobenzoic acid (PABA), ethyl-PABA and butyl-PABA. PABA is the most hydrophilic compound and its lipophilicity increases with increasing ester chain length. The diffusion profiles of all three model compounds across 12 $\mu \mathrm{m}$ thick lipid membranes closely resemble those of human stratum corneum [27]. Exclusion of EOS from the lipid mixture revealed a reduced barrier function of the stratum corneum substitute, demonstrating that CER1 is not only very important for a proper skin lipid phase behaviour, but also for the skin barrier function (see Fig. 3B). This indicates that the reduced barrier function in psoriatic and LI skin might partially be caused by a reduction in EOS in stratum corneum. In future we will use this excellent tool to study the relation between lipid organisation and lipid composition into more details to determine the role the various lipids play in the skin barrier function.

\section{CONCLUSIONS}

Although impressive progress has been made in elucidating the lipid organisation in stratum corneum, essential information is still missing. For example the lipid lamellar organisation is still under debate as the localisation of the lipids within the LPP and SPP is not fully understood. Furthermore, only limited information is available on the 
lipid organisation and composition in stratum corneum of diseased skin. However, this unequivocally stresses that there is a need to explore this scientific field in more detail.

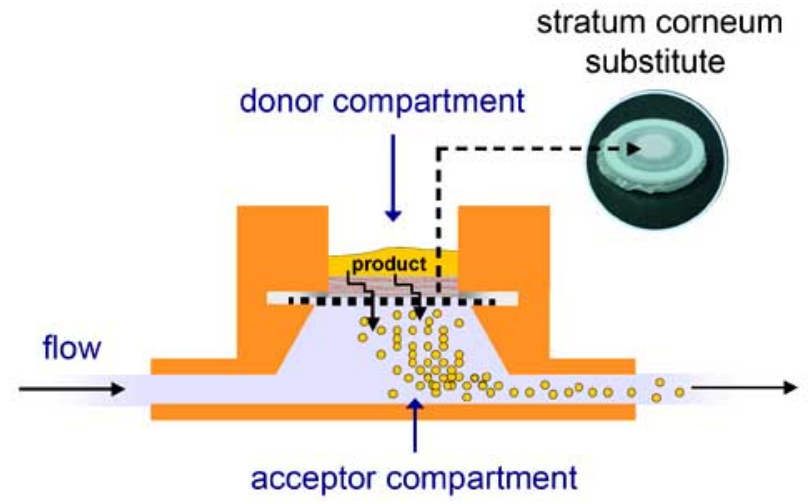

Fig. (3A). Diffusion studies can be performed across a lipid membrane mimicking the barrier properties and lipid organisation of human stratum corneum. These membranes are referred to as the stratum corneum substitute.

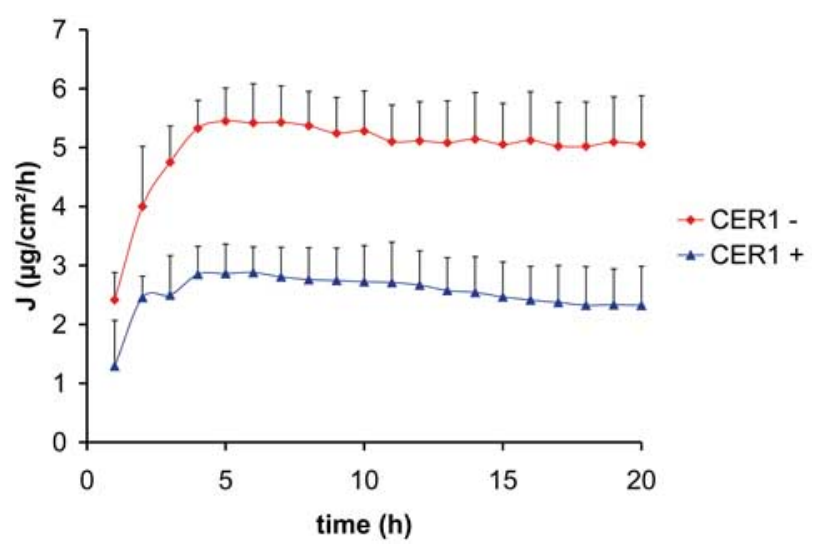

Fig. (3B). In the absence of EOS, (referred to as CER1) an increase in the flux of ethyl-PABA is observed, indicating a reduced barrier function.

\section{REFERENCES}

[1] Harding CR, Watkinson A, Rawlings AV. Dry skin moisturisation and corneodesmolysis. Int J Cosm Sci 2000; 22: 21-52.

[2] Rawlings AV. Trends in stratum corneum research. Int J Cosm Sci 2003; 25: 1-33.

[3] Wertz PW, Downing DT. In: LA Goldsmith, Ed. Physiology. biochemistry and molecular biology of the skin. $2^{\text {nd }}$ ed. New York, Oxford University Press 1991: pp. 205-36.

[4] Schurer NY, Elias PM. The biochemistry and function of stratum corneum. Adv Lipid Res 1991; 24: 27-54.

[5] Weerheim A, Ponec M. Determination of stratum corneum lipid profile by tape stripping in combination with high-performance thin-layer chromatography. Arch Derm Res 2001; 292: 191-9.

[6] Robson KJ, Stewart ME, Michelsen S, Lazo ND, Downing DT. 6Hydroxy-4-sphingenine in human epidermal ceramides. J Lipid Res 1994; 35: 2060-8.
[7] Stuart ME, Downing DT. 6-Hydroxy-4-sphingenine in human epidermal ceramides. J Lipid Res 1999; 40: 1434-39.

[8] Ponec M, Lankhorst P, Weerheim A, Wertz P. New acylceramide in native and reconstructed epidermis. J Invest Dermatol 2003; 120: 5818.

[9] Musakawa Y, Narita H, Shimizu E, et al. Characterization of overall ceramide species in human stratum corneum. J Lipid Res 2008; 49: 1466-67.

[10] Motta SM, Monti M, Sesana S, Caputo R, Carelli S, Ghidoni R. Ceramide composition of psoriatic scale. Biochim Biophysic Acta 1993; 1182: 147-51.

[11] Bouwstra JA, Gooris GS, van der Spek JA, Bras W. Structural investigations on human stratum corneum by small angle X-ray scattering. J Invest Dermatol 1991; 97: 1005-12.

[12] Bouwstra JA, Gooris GS, Salomons-de Vries MA, van der Spek JA, Bras W. Structure of human stratum corneum as function of temperature and hydration: A wide angle $\mathrm{x}$-ray diffraction study. Int $\mathrm{J}$ Pharm 1992; 84: 205-16.

[13] Elias PM, Bonar L, Grayson S, Baden HP. X-ray diffraction analysis of stratum corneum membrane couplets. J Invest Dermatol 1983; 80: 21314.

[14] Garson J-C, Doucet J, Lévêque J-L, Tsoucaris GJ. Oriented structure in human stratum corneum revealed by X-ray diffraction. J Invest Dermatol 1991; 96: 43-9.

[15] Pilgram GSK, Engelsma-van Pelt AM, Bouwstra JA, Koerten HK. Electron diffraction provides new information on human stratum corneum lipid organization studied in relation to depth and temperature. J Invest Dermatol 1999; 113: 403-9.

[16] Pilgram GSK, Vissers DCJ, van der Meulen H, et al. Aberrant lipid organization in stratum corneum of patients with atopic dermatitis and lamellar ichthyosis. J Invest Dermatol 2001; 117: 710-7.

[17] Gay CL, Guy RH, Golden GM, Mak VHW, Francoeur M. Characterization of low-temperature lipid transitions in human stratum corneum. J Invest Dermatol 1994; 103: 233-9.

[18] Damien F, Boncheva M. The extent of the orthorhombic phase in stratum corneum determines the barrier efficiency of human skin in vivo. J Invest Dermatol 2010; 130: 611-4.

[19] Bouwstra JA, Gooris GS, Dubbelaar FER, Ponec M. Phase behaviour of lipid mixtures based on human ceramides: coexistence of crystalline and liquid phases. J Lipid Res 2001; 42: 1759-70.

[20] Bouwstra JA, Gooris GS, Dubbelaar FER, Ponec M. pH and cholesterol sulfate and fatty acids affect the stratum corneum lipid organisation. J Invest Dermatol Symp Proc 1998; 3: 69-74.

[21] Bouwstra JA, Gooris GS, Cheng K, Weerheim A, Bras W, Ponec M. Phase behaviour of isolated skin lipids. J Lip Res 1996; 37: 999-1011.

[22] Motta S, Monti M, Sesana S, Caputo R, Carelli S, Ghidoni RL. Ceramide composition of the psoriatic scale. Biochim Biophys Acta 1993; 1182: 147-51.

[23] Lavrijsen APM, Bouwstra JA, Gooris GS, Boddé HE, Ponec M. Reduces skin barrier function parallels. Abnormal stratum corneum lipid organisation in patients with lamellar ichtyosis. J Invest Dermatol 1995; 105: 619-24.

[24] Schreiner V, Gooris GS, Pfeifer S, et al. Barrier characteristics of different human skin types investigated with $\mathrm{x}$-ray diffraction, lipid analysis and electron microscopy imaging. J Invest Dermatol 2000; 114: 654-60.

[25] de Jager MW, Gooris GS, Ponec M, Bouwstra JA. Lipid mixtures prepared with well-defined synthetic ceramides closely mimic the unique stratum corneum lipid phase behavior. J Lipid Res 2005; 46: 2649-56.

[26] Janssens M, Gooris GS, Bouwstra JA. Infrared spectroscopy studies of mixtures prepared with synthetic ceramides varying in head group architecture: coexistence of liquid and crystalline phases. Biochim Biophys Acta Biomembr 2009; 1788: 732-42.

[27] de Jager MW, Groenink W, Bielsa i Guivernau R, et al. A novel in vitro percutaneous penetration model: evaluation of barrier properties with $\mathrm{P}$ Aminobenzoic acid and two of its derivatives. Pharm Res 2006; 23: 951-60. 